\title{
Occlusal Adjustment
}

National Cancer Institute

\section{Source}

National Cancer Institute. Occlusal Adjustment. NCI Thesaurus. Code C62300.

Reshaping a tooth surface to modify the dental occlusion. 